\title{
Evidence for a Double Dissociation of Articulatory Rehearsal and Non-Articulatory Maintenance of Phonological Information in Human Verbal Working Memory
}

\author{
Sarah Trost Oliver Gruber \\ Department of Psychiatry and Psychotherapy, Center for Translational Research in Systems Neuroscience and \\ Clinical Psychiatry, Georg August University, Goettingen, Germany
}

\begin{abstract}
Key Words
Brain lesion - Broca's area - Lesion deficit approach •

Phonological store $\cdot$ Articulatory suppression - Verbal

rehearsal · Frontopolar cortex $\cdot \mathrm{fMRI} \cdot$ Neuroimaging ·

Experimental neuropsychology
\end{abstract}

\begin{abstract}
Objective: Recent functional neuroimaging studies have provided evidence that human verbal working memory is represented by two complementary neural systems, a left lateralized premotor-parietal network implementing articulatory rehearsal and a presumably phylogenetically older bilateral anterior-prefrontal/inferior-parietal network subserving non-articulatory maintenance of phonological information. In order to corroborate these findings from functional neuroimaging, we performed a targeted behavioural study in patients with very selective and circumscribed brain lesions to key regions suggested to support these different subcomponents of human verbal working memory. Methods: Within a sample of over 500 neurological patients assessed with high-resolution structural magnetic resonance imaging, we identified 2 patients with corresponding brain lesions, one with an isolated lesion to Broca's area and the
\end{abstract}

other with a selective lesion bilaterally to the anterior middle frontal gyrus. These 2 patients as well as groups of agematched healthy controls performed two circuit-specific verbal working memory tasks. In this way, we systematically assessed the hypothesized selective behavioural effects of these brain lesions on the different subcomponents of verbal working memory in terms of a double dissociation. $\boldsymbol{R e}$ sults: Confirming prior findings, the lesion to Broca's area led to reduced performance under articulatory rehearsal, whereas the non-articulatory maintenance of phonological information was unimpaired. Conversely, the bifrontopolar brain lesion was associated with impaired non-articulatory phonological working memory, whereas performance under articulatory rehearsal was unaffected. Conclusion: The present experimental neuropsychological study in patients with specific and circumscribed brain lesions confirms the hypothesized double dissociation of two complementary brain systems underlying verbal working memory in humans. In particular, the results demonstrate the functional relevance of the anterior prefrontal cortex for non-articulatory maintenance of phonological information and, in this way, provide further support for the evolutionary-based functional-neuroanatomical model of human working memory.

Copyright @ 2012 S. Karger AG, Basel

\section{KARGER}

Fax +4161306 1234

E-Mail karger@karger.ch

www.karger.com (c) 2012 S. Karger AG, Basel

0302-282X/12/0653-0133\$38.00/0

Accessible online at:

www.karger.com/nps
Dr. Oliver Gruber, MD

Department of Psychiatry and Psychotherapy

Georg August University

DE-37075 Goettingen (Germany)

Tel. +49 551391 2289, E-Mail ogruber@gwdg.de 


\section{Introduction}

Human working memory is conceptualized as a set of linked and interacting processing components that allow temporary storage and simultaneous manipulation of information in the brain. These processing components play a key role for higher cognitive functions such as language, planning, problem solving and reasoning [1].

During the last few decades, the three-component model provided by Baddeley and Hitch $[1,2]$ has been the most influential theoretical concept of working memory $[3,4]$. This three-component model comprises a control system of limited attentional capacity, the so-called central executive, which is assisted by two subsidiary storage systems, namely the visuospatial sketch pad and the phonological loop [5]. While it is still a matter of debate whether spatial working memory can be further subdivided into active rehearsal and passive storage components [6], this has been well established for verbal working memory in humans [7]. Numerous functional neuroimaging studies have been conducted during the last few years in order to investigate the neural implementation of human working memory. In accordance with the cognitive model provided by Baddeley and Hitch $[1,2]$, different brain systems have been found to underlie verbal and visuospatial working memory processes $[8,9]$. Furthermore, a recent series of functional neuroimaging studies in healthy human subjects has provided compelling evidence that verbal working memory in humans is supported by two different brain systems [7-9]. A mainly left-hemispheric premotor-parietal system including Broca's area, the left lateral premotor cortex and intraparietal cortex as well as the right cerebellum is involved in articulatory rehearsal, i.e. 'inner speech' $[7,10-12]$, whereas a bilateral prefrontoparietal system comprising the cortex along anterior parts of the intermediate frontal sulcus, the inferior parietal lobules and the anterior cingulate cortex has repeatedly been demonstrated to subserve a non-articulatory mechanism for maintaining phonological information, which corresponds to the concept of the 'inner ear' $[7,9,13]$.

Based on these empirical data and in consideration of the functional-neuroanatomical homologies observed in monkeys, an evolutionary-based functional-neuroanatomical model of working memory has been proposed according to which human working memory consists of two different and at least partially dissociable neural systems that interact with each other [3]. A presumably phylogenetically older, multimodal working memory system, which is also present in non-human primates, is implemented by several domain-specific prefrontoparietal and prefrontotemporal networks [14]. On the other hand, a second system, which probably developed later in the context of the evolution of language, is supported by mostly left-hemispheric speech areas and mediates explicit verbal rehearsal [15]. This model appears promising in that it may not only offer new explanations for many behavioural, neuropsychological and neuroimaging findings in human subjects, but it also permits the harmonization of the conflicting working memory models that were derived from human as well as animal research $[2,15]$.

While functional neuroimaging permits the investigation of regional brain activity changes that are associated with specific cognitive processes or behaviours, only lesion studies allow to determine the functional relevance of a certain brain area for the task that has to be performed. Therefore, it is necessary to combine these two methodological approaches in the attempt to establish valid models of brain-behaviour relationships. With regard to the above-mentioned two neurofunctional systems, data from lesion studies in brain-damaged patients seem to suggest that the prefrontal cortex might not be necessary for delay task performance, i.e. working memory [16]. In particular, these reports seem to indicate that the cortex along the anterior intermediate frontal sulcus, which showed reliable and reproducible activation in functional neuroimaging studies during the non-articulatory maintenance of phonological information, might not be functionally relevant for such maintenance processes in working memory. However, the brain lesions observed in individual patients are usually not restricted to functionally homogeneous areas of the brain which may account for misleading results of behavioural studies in such patients. The same is true for group lesion studies in which an artificial overlap of individual brain lesions is used to infer functional relevance, thereby not taking into consideration the high functional heterogeneity of brain areas and the concept of functional degeneracy [17, 18]. For this reason, in order to judge the functional relevance of circumscribed brain areas, it may certainly be more informative to assess rare individual cases of patients with clearly isolated lesions to the brain regions of interest.

Therefore, in order to further corroborate the dissociability of the two functional brain systems mentioned above and to demonstrate the functional relevance of some of the brain areas involved, we searched and found in a set of over 500 neurological patients with brain lesions 2 patients with isolated focal lesions to brain areas involved in the two neurofunctional systems. In particular, we were interested in 1 patient who showed an isolated left-sided lesion in Broca's area, and in another patient who exhibited a bi- 
lateral lesion along anterior parts of the middle frontal gyrus (along the intermediate frontal sulcus). According to the functional-neuroanatomical model outlined above, these patients should reveal specific deficits to either the articulatory or the non-articulatory maintenance component of verbal working memory. The aim of this study was first to provide this additional behavioural evidence for the functional relevance of the anterior middle frontal gyrus for non-articulatory maintenance of phonological information, and second to assess possible compensatory activation of this second neurofunctional system by using fMRI in the patient with the lesion to Broca's area.

\section{Materials and Methods}

\section{Subjects}

Two neurological patients with isolated brain lesions, one with a lesion to Broca's area due to infarction (P403; fig. 1) and the other with a bilateral lesion to the anterior middle frontal gyrus due to trauma (P300; fig. 2), as well as two separate groups of 3 healthy male controls (each matched for age, handedness and education to the respective patient) participated in this study. A further control group $(n=9)$ for patient P300 was additionally recruited to consolidate the $95 \%$ confidence interval concerning verbal working memory performance rates.

The first neurological patient (P403; fig. 1) selected was a 70 -year-old, right-handed man with a history of hypercholesterolaemia, nicotine abuse of 25 pack years and suspicion of hyperfibrinogenaemia, who had first been admitted to hospital because of acute aphasia about 5 months prior to our investigation. At this time, patient $\mathrm{P} 403$ was diagnosed with non-fluent, anomic and expressive aphasia and agraphia combined with minimal rightsided facial paralysis. Computed brain tomography and MRI revealed a left-sided ischaemic infarction of Broca's area (middle cerebral artery), most likely of autochthone thrombotic origin according to the patient's cardiovascular risk profile. Up to the time of our investigation, patient $\mathrm{P} 403$ showed no more relevant motor or cognitive deficits and his verbal everyday communication was unimpaired. There were only slight residual deficits in speech production concerning abstract terms. Patient P403 had passed 13 years of education.

The second neurological patient (P300; fig. 2) included in the study was a 40-year-old man, right-handed, who had also passed 13 years of education. Patient P300 had fallen off a scaffolding with a height of $4 \mathrm{~m}$ at the age of 21 during his work on a construction area. He was diagnosed with severe closed traumatic brain injury, namely bilateral frontopolar haemorrhagic brain contusion resulting in brain tissue necrosis of the bifrontopolar cortex in the long term. Since his accident, patient P300 presented with lacking motivation and destitute social skills in his family, while he was still able to continue working in his familiar environment of the construction business. Neuropsychological testing revealed mild deficits in memory and executive functioning, but unimpaired information processing speed and sustained attention at the time of our investigation.

Double Dissociation in Human Verbal Working Memory

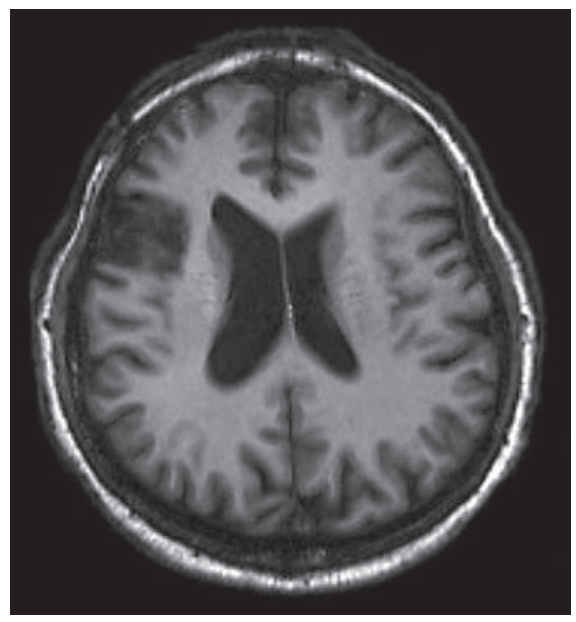

Fig. 1. Structural MRI of patient P403 illustrating the lesion to Broca's area (axial view).

All subjects had given written informed consent prior to the experiment. All participants were trained to perform the experimental verbal item recognition task.

\section{Behavioural Experiment}

Experimental Procedure

All subjects performed two variants of a verbal item recognition task after being instructed to use two different cognitive strategies of maintaining and memorizing verbal information (e.g. verbal rehearsal, 'inner speech' vs. phonological maintenance, 'inner ear'; for details see below) [7-9, 19-21].

Subjects were seated approximately $65 \mathrm{~cm}$ from a computer monitor and were pretrained for about $30 \mathrm{~min}$ to successfully complete the two variants of the verbal item recognition task. In both variants of this working memory task, in each trial 4 different letters that were randomly taken out of a set of 8 phonologically similar letters (in German: B, C, D, E, G, P, T and W) were visually presented on the monitor for $1 \mathrm{~s}$, followed by a delay of 4 s during which a fixation cross was displayed. Then, a single probe letter was presented on the screen for $1 \mathrm{~s}$ and subjects were expected to signal whether this probe letter matched 1 of the 4 priorly presented target letters or not by pressing one of two response buttons (left/right button press with the index/middle finger of the right hand). Trials were separated by a 1-second fixation period which resulted in an overall maximal response time of $2 \mathrm{~s}$. Half of the probe letters matched the target letters, the other half did not. If the 4 target letters were presented in uppercase, the probe letter was lowercase (and vice versa) in order to prevent subjects from using purely visual strategies for matching. This general trial structure was identical in both variants of the verbal working memory task.

Before each trial, a visual cue instructed the subjects to either read, memorize and maintain the 4 target letters using one of two different strategies (task conditions, for details see below) or to perform a control task. As control task, subjects were instructed to silently read the 4 target letters without memorizing and maintaining them, and then to judge whether the subsequently pre- 

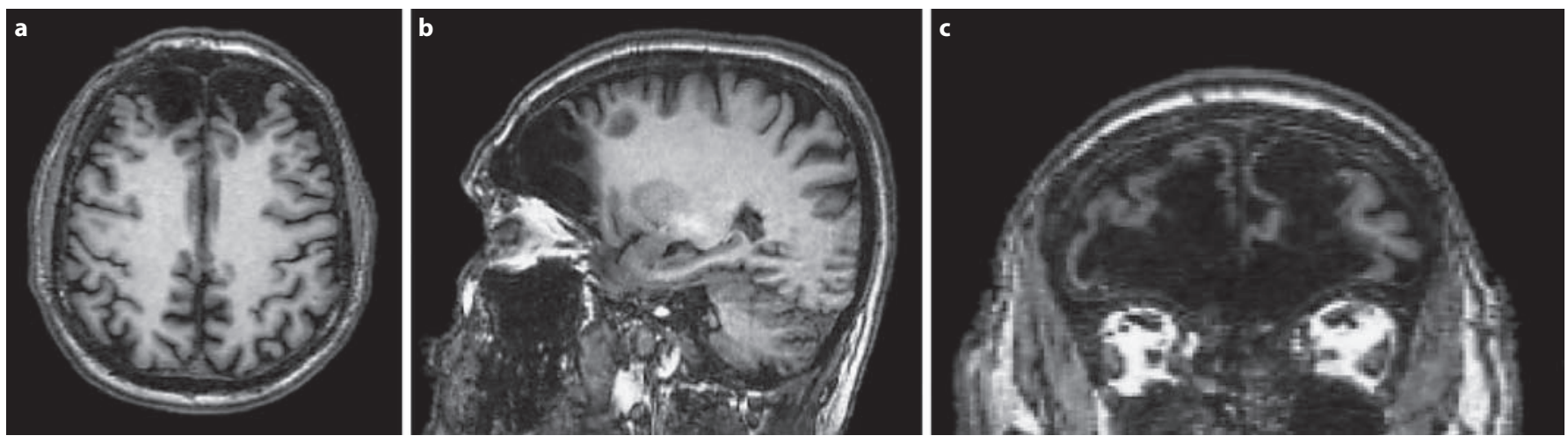

Fig. 2. Structural MRI of patient $P 300$ depicting the exact location of the bifrontopolar brain lesion. a Axial, b sagittal and c coronal view.

General trial structure:
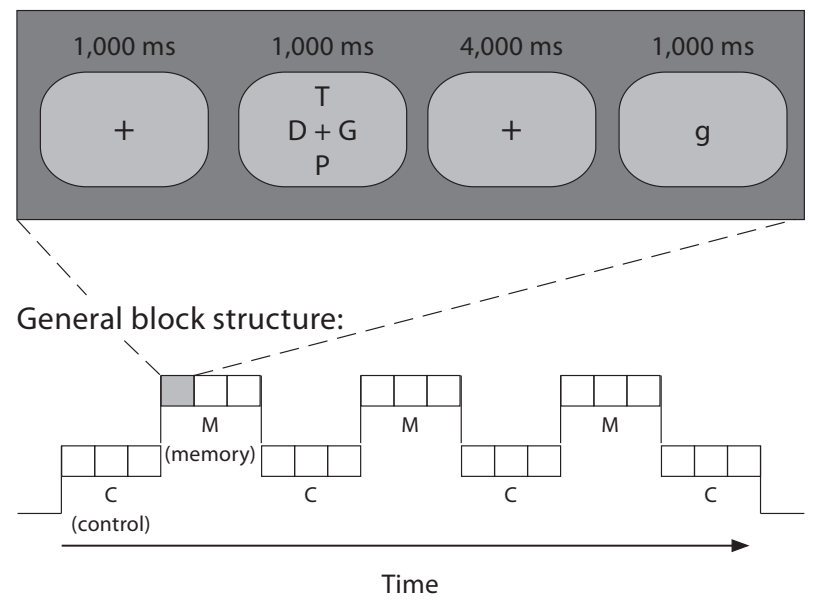

Fig. 3. Experimental design (block design). Patients and controls performed a verbal item recognition task (M; two different versions) in cued alternation with a letter case judgment task (C). Different blocks varied with respect to the 4-second delays, which were either unfilled (verbal rehearsal) or filled with silent counting (articulatory suppression). See Materials and Methods for details.

sented single probe letter was uppercase (left button) or lowercase (right button). In the control task, this probe letter was uppercase in half of the trials, and in the other half lowercase, unpredictable for the subjects (fig. 3).

The two verbal item recognition (working memory) task variants introduced in this study essentially differed in the strategies the participants had to apply to memorize the 4 target letters. In one variant, subjects were instructed to use articulatory rehearsal (silently and repeatedly subvocalizing the 4 presented letters, 'inner speech') in order to actively maintain the presented letters, whereas in the other variant of the task, they had to maintain the verbal information without using the rehearsal mechanism (pho- nological maintenance, 'inner ear'). The latter strategy was forced by an articulatory suppression procedure, i.e. the participants had to silently count ' $1,2,3,4,1,2,3, \ldots$ ' in a repetitive and rapid manner throughout the delay after the presentation of the target letters in order to prevent them from making use of the articulatory rehearsal strategy.

Thus, the two task variants (including the corresponding control trials, respectively) varied with respect to the 4-second delays, which were either unfilled (articulatory rehearsal task) or filled with tones that paced silent counting (phonological maintenance task under articulatory suppression). Rhythmic $4,000-\mathrm{Hz}$ tones with a repetition frequency of $300 \mathrm{~ms}$ set the pace of the silent counting. During articulatory suppression, this pace ensured high demands on subvocalization processes, which strongly interfere with verbal rehearsal of the letters $[7,9]$.

Overall, 54 memory trials were presented for each of the task conditions which were arranged in a counterbalanced order. For task presentation on the computer monitor, we used the computer software package Presentation (Neurobehavioral Systems, Albany, N.Y., USA).

Statistical Analysis of Behavioural Data

Statistical analyses of the behavioural data were performed using SPSS for Windows 16.0 (SPSS Inc., Chicago, Ill., USA). Performance rates in the two working memory tasks, as well as in the control conditions, were determined for the patients and were compared to performance rates in the groups of healthy controls. For this, the $95 \%$ confidence intervals of the mean performance rates of the respective control group were determined (table 1) and served as a reference value for the patient's performance.

\section{fMRI Experiment}

Experimental Design and Data Acquisition

A 3.0-tesla MRI scanner (Bruker Medspec 30/100) with a circularly polarized head coil was used to first obtain a high-resolution structural scan of the patients. Afterwards, three runs of 518 gradient echo-planar image volumes each were acquired $(\mathrm{TR}=$ $2 \mathrm{~s}, \mathrm{TE}=40 \mathrm{~ms}$, flip angle $=90^{\circ}$, number of slices $=16$, voxel size $=$ $3 \times 3 \times 5 \mathrm{~mm}^{3}$, distance factor $=0.2$ ). Functional image volume acquisition was synchronized with stimulus presentation by means of ERTS (Experimental Run Time System, version 3.11, 
Table 1. Working memory performance rates of the two neurological patients (P403 and P300) and healthy control subjects ( $\mathrm{n}=3$ and $\mathrm{n}=9$, respectively) with respect to two different working memory tasks (verbal rehearsal vs. phonological maintenance under articulatory suppression)

\begin{tabular}{|c|c|c|c|c|c|}
\hline Working memory task, percentage correct & P403 & P300 & $\begin{array}{l}\text { Healthy control } \\
\text { subjects (vs. P403) } \\
(\mathrm{n}=3) \text { (original) }\end{array}$ & $\begin{array}{l}\text { Healthy control } \\
\text { subjects (vs. P300) } \\
(\mathrm{n}=3) \text { (original) }\end{array}$ & $\begin{array}{l}\text { Healthy control } \\
\text { subjects (vs. P300) } \\
(n=9) \text { (post hoc) }\end{array}$ \\
\hline $\begin{array}{l}\text { Phonological maintenance } \\
\quad 95 \% \text { confidence interval (mean } \pm 2 \times \mathrm{SD})\end{array}$ & 74 & 61 & $\begin{array}{l}81.67 \pm 6.11 \\
69.45-93.89\end{array}$ & $\begin{array}{l}79.00 \pm 7.21 \\
64.58-93.42\end{array}$ & $\begin{array}{l}88.89 \pm 5.84 \\
77.21-100.00\end{array}$ \\
\hline
\end{tabular}

$\mathrm{SD}=$ Standard deviation.

BeriSoft Cooperation, Frankfurt am Main, Germany). The stimulation protocol was the same as in the behavioural experiment described above.

\section{fMRI Data Analysis}

The fMRI data were analysed using the software package LIPSIA [22]. First, the functional images were corrected for motion artefacts. Then, a correction for slice-time acquisition differences was performed using a sinc interpolation algorithm. Afterwards, the 2-dimensional MDEFT and echo-planar image- $\mathrm{T}_{1}$ slices geometrically aligned with the functional slices were coregistered with the high-resolution 3-dimensional reference $\mathrm{T}_{1}$ data set. Rotational and translational parameters computed for this registration were stored in a transformation matrix. This matrix was directly applied to the functional data resulting in a coregistration with the individual 3-dimensional anatomical data set. Finally, the data set was spatially smoothed using Gaussian filter kernels with a $\sigma$ of 0.5 (for single subject analysis).

For statistical analysis, the alternating task periods were modelled using a delayed box-car reference vector accounting for the delay of the BOLD (blood oxygen level-dependent) response. Significantly activated pixels were searched for using the general linear model approach for time series data. Results are reported for brain activations that reached a voxelwise significance level of $\mathrm{p}<0.001$, uncorrected.

\section{Results}

\section{Behavioural Data}

As we had hypothesized, the patient with the lesion to Broca's area exhibited a marked deficit in the articulatory rehearsal task variant. His verbal working memory performance under rehearsal conditions lay outside the 95\% confidence interval of the age-matched control group (table 1; fig. 4), while his performance in the non-articulatory version of the task (articulatory suppression) was clearly within the $95 \%$ confidence interval of the control group (table 1; fig. 4).

Double Dissociation in Human Verbal Working Memory
The lesion to Broca's area was thus associated with a selective deficit of the articulatory rehearsal mechanism of verbal working memory leading to an elimination of the typical articulatory suppression effect [7-9]. This means that performance rates of patient $\mathrm{P} 403$ in the verbal rehearsal condition were even below his performance rates under articulatory suppression (table 1), which stands in contrast to the group of healthy controls that showed the typical articulatory suppression effect in terms of lowered performance rates under articulatory suppression as compared to the verbal rehearsal condition (table 1; fig. 4).

As predicted, in the patient with the bifrontopolar lesion (P300), exactly the opposite pattern of deficits was observed. Working memory performance of this patient under articulatory suppression was clearly impaired and lay outside the $95 \%$ confidence interval of the mean values of the age-matched control group $(\mathrm{n}=3)$. By contrast, in the articulatory rehearsal version of the task, the patient's performance did not differ from that of the healthy controls (table 1; fig. 5). Following a reviewer's suggestion, we additionally compared the performance rates of patient P300 under articulatory suppression with a further, larger group of matched healthy controls (matched for age, handedness, gender and years of education; $n=9$ ). Again, the performance rates of patient P300 in the articulatory suppression task condition lay outside the $95 \%$ confidence interval of this larger control group, i.e. patient P300 showed a selective deficit of the non-articulatory mechanism of verbal working memory, while his performance of the verbal rehearsal task was unimpaired (table 1).

\section{Functional Imaging Data}

The patient with the lesion to Broca's area, P403 (fig. 6), revealed significant perilesional brain activity and, additionally, bilateral activations of anterior prefrontal (and 


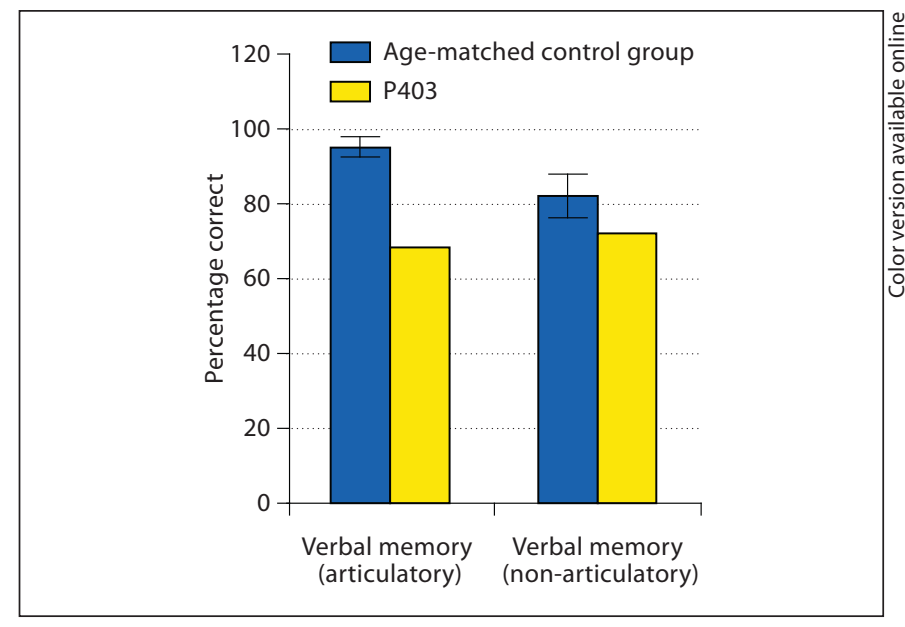

Fig. 4. Performance of the patient (P403) with the lesion to Broca's area during articulatory rehearsal conditions (left side) and during non-articulatory maintenance of phonological information (right side) compared to age-matched controls $(n=3)$. Performance in the non-articulatory version of the task lies within the $95 \%$ confidence interval of the age-matched control group.

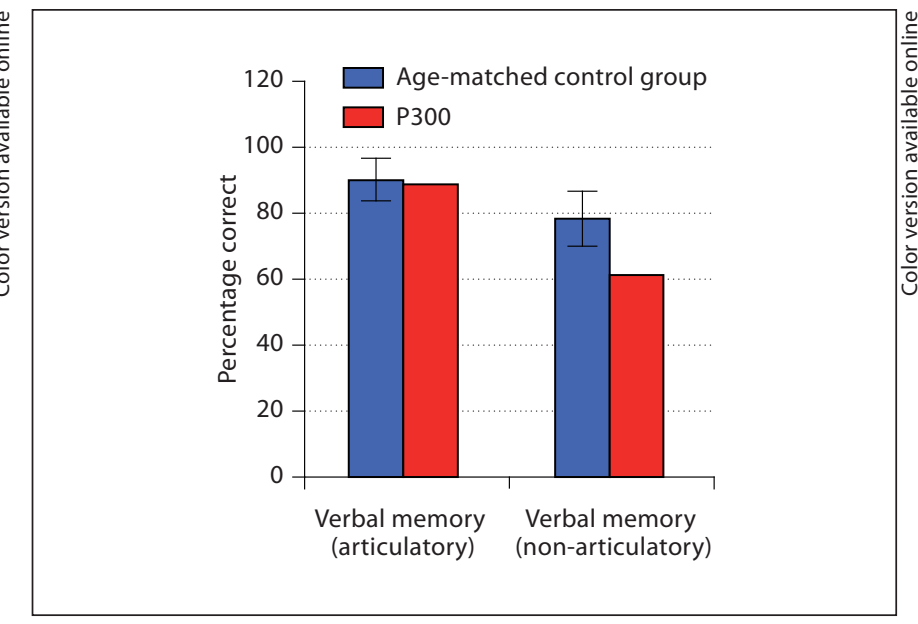

Fig. 5. Performance of the patient (P300) with the bifrontopolar lesion during articulatory rehearsal conditions (left side) and during non-articulatory maintenance of phonological information (right side) compared to age-matched controls $(n=3)$. Impaired performance under articulatory suppression and unaffected performance in the articulatory version of the task as compared to age-matched controls.
Fig. 6. Brain activations of the patient with the lesion to Broca's area. Significant perilesional brain activity (a) and, additionally, bilateral activations of the anterior prefrontal cortex $(\mathbf{a}, \mathbf{b})$ even under articulatory rehearsal conditions. $\mathrm{p}<0.001$, uncorrected.

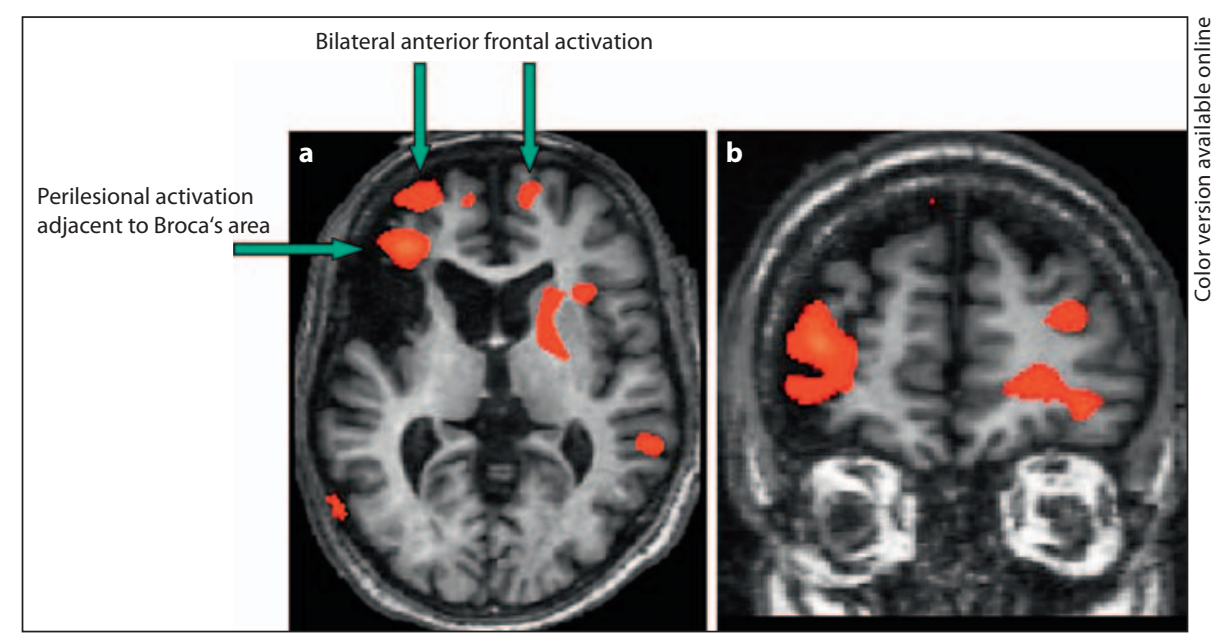

inferior parietal) brain areas even under articulatory rehearsal conditions (fig. 6), which may explain the partially preserved performance in the articulatory version of the verbal working memory task.

\section{Discussion}

A large series of fMRI studies clearly indicates that human verbal working memory relies on two different neurofunctional systems, one system consisting of mostly left-hemispheric speech areas and mediating articulatory rehearsal, and the other one represented by several domain-specific prefrontoparietal and prefrontotemporal networks that underlie the non-articulatory maintenance of different types of information (visuospatial information, visual object features, auditory-phonological information) [7-9]. The present study aimed at providing converging evidence from the behaviour of patients with a focal brain lesion to confirm the dissociability of these two neurofunctional systems. In particular, the study assessed the functional relevance of the anterior prefrontal 
cortex along the middle frontal gyrus for the non-articulatory maintenance of phonological information in working memory. Within a sample of over 500 neurological patients assessed with structural MRI, we identified 2 patients with very selective and circumscribed brain lesions to either Broca's area, which has been found in numerous fMRI studies to be activated during articulatory rehearsal, or bilaterally to the frontopolar cortex including the anterior middle frontal gyrus, which has been repeatedly demonstrated to be activated during the non-articulatory maintenance of phonological information [7-9]. In fact, the behavioural results obtained in these 2 patients demonstrate a clear double dissociation between structure and function. While the lesion to Broca's area led to a reduced performance under articulatory rehearsal, but not during non-articulatory maintenance of phonological information, the bifrontopolar lesion was associated with unaffected performance under articulatory rehearsal conditions, but with severely impaired non-articulatory maintenance of phonological information. These data clearly confirm the functional significance of Broca's area and the bilateral anterior middle frontal gyrus for articulatory rehearsal and non-articulatory maintenance of phonological information in verbal working memory, respectively.

While the present findings are consistent with the results of multiple functional neuroimaging studies of working memory $[7,9,10,12,20]$, other recent behavioural studies in patients with brain lesions seem to suggest that particularly the lateral prefrontal cortex might not essentially contribute to or might not be necessary for delay task performance, but rather inferior parietal regions including the supramarginal gyrus $[16,23,24]$. Besides some internal inconsistencies between the data reported from these lesion and fMRI studies, it is also important to point out that, for example, none of the 7 patients assessed in a recent study [16] had a lesion in the anterior prefrontal subregion that has been implicated in non-articulatory maintenance of phonological information by many fMRI studies [7-9] and also by the present lesion study. For these reasons, the present finding and confirmation of the functional role of the anterior middle frontal gyrus in the non-articulatory component of verbal working memory is still compatible and can be easily reconciled with the (negative) results of such prior behavioural studies in brain-lesioned patients. In this sense, our findings demonstrate the necessity to use well-defined experimental paradigms with high process specificity as well as exact and precise neuroanatomical definitions in order to be able to establish clear brain-behaviour relationships. Clearly, group lesion studies, in which an

Double Dissociation in Human Verbal Working Memory artificial overlap of individual brain lesions is used to infer functional relevance, do not fulfil these strict methodological criteria. As such group lesion studies are unable to account for the high functional heterogeneity of brain areas in individual patients and for the concept of functional degeneracy [18], they are prone to obscure existing brain-behaviour relationships, on the one hand, and to misattribute general dysfunctions of complex networks of interacting brain regions to the overlapping intersection of lesions in the whole group of patients.

Another alternative approach to investigate the effects of isolated brain lesions is represented by transcranial magnetic stimulation (TMS) [25]. As a well-tolerated, non-invasive technique, TMS allows the stimulation or functional disruption of cortical areas through the intact scalp, limited in time. Thus, a 'virtual' brain lesion can be produced to investigate local brain function with high temporal resolution. However, the duration of a TMS-induced 'virtual' lesion is limited and stimulation intensities are varying with the distance of the target brain region from the TMS coil. Beyond that, TMS still lacks exact focal accuracy and exhibits poor spatial resolution [25-28]. Recent experimental studies have introduced a combined use of TMS with other brain mapping techniques in order to compensate for these methodologic weaknesses thereby providing a new promising set of tools for future research [28].

While our finding of the functional relevance of Broca's area for the articulatory rehearsal mechanism merely represents a replication of prior findings [29], a second objective of our study was to find evidence for possible compensatory functional activation of the anterior middle frontal gyrus in the patient with the lesion to Broca's area. (It is important to note that in this chronic patient, language functions had already recovered to a large part. Nevertheless, this patient exhibited a clear deficit in the articulatory rehearsal task.) For this, we used fMRI in this patient during performance of the articulatory rehearsal task. Besides a perilesional activation anterior to the lesion in Broca's area, we indeed observed additional bilateral activations of the anterior middle frontal gyrus (fig. 6). In a previous fMRI study, it could be shown that when exactly the same experimental task is performed by healthy subjects, the anterior middle frontal gyrus becomes actively suppressed by brain activity in the articulatory rehearsal system [13]. Therefore, our present finding may be taken as first, although preliminary evidence that a lesion to Broca's area may lead to a disinhibition of the subordinate network subserving non-articulatory maintenance of phonological information, and that this may partially compensate for the functional consequences of the lesion. 
To conclude, the present findings from a combination of functional neuroimaging and behavioural studies in patients with selective and clearly circumscribed brain lesions are able to confirm the hypothesized double dissociation of the two neurofunctional systems underlying the articulatory and the non-articulatory maintenance of phonological information in working memory. In this way, the present results provide further support for the evolutionary-based functional neuroanatomical model of human working memory. Last but not least, the results provide clear evidence that the cortex along anterior parts of the middle frontal gyrus is functionally relevant for the non-articulatory mechanism of verbal working memory in humans.

\section{Acknowledgments}

We thank the Max Planck Institute for Human Cognitive and Brain Sciences and in particular D. Yves von Cramon for providing the fMRI facilities that made this work possible. We thank Dr. Stephan Konrad for help in data collection.

\section{References}

1 Baddeley A: Working memory. Science 1992; 255:556-559.

2 Baddeley AD, Hitch GJ: Working memory; in Bower GA (ed): The Psychology of Learning and Motivation: Advances in Research and Theory. New York, Academic Press, 1974, vol 8, pp 47-89.

-3 Gruber O, Goschke T: Executive control emerging from dynamic interactions between brain systems mediating language, working memory and attentional processes. Acta Psychol (Amst) 2004;115:105-121.

4 Buchsbaum BR, D’Esposito M: The search for the phonological store: from loop to convolution. J Cogn Neurosci 2008;20:762-778.

5 Baddeley A: Working memory: looking back and looking forward. Nat Rev Neurosci 2003;4:829-839.

6 Washburn DA, Astur RS: Nonverbal working memory of humans and monkeys: rehearsal in the sketchpad? Mem Cognit 1998;26:277-286.

7 Gruber O: Effects of domain-specific interference on brain activation associated with verbal working memory task performance. Cereb Cortex 2001;11:1047-1055.

$>8$ Gruber O, von Cramon DY: Domain-specific distribution of working memory processes along human prefrontal and parietal cortices: a functional magnetic resonance imaging study. Neurosci Lett 2001;297:29-32.

-9 Gruber O, von Cramon DY: The functional neuroanatomy of human working memory revisited. Evidence from 3-T fMRI studies using classical domain-specific interference tasks. Neuroimage 2003;19:797-809.

10 Paulesu E, Frith CD, Frackowiak RS: The neural correlates of the verbal component of working memory. Nature 1993;362:342-345.

-11 Fiez JA, Raichle ME, Balota DA, Tallal P, Petersen SE: PET activation of posterior temporal regions during auditory word presentation and verb generation. Cereb Cortex 1996;6:1-10.

$\checkmark 12$ Jonides J, Schumacher EH, Smith EE, Koeppe RA, Awh E, Reuter-Lorenz PA, Marshuetz C, Willis CR: The role of parietal cortex in verbal working memory. J Neurosci 1998;18:5026-5034.
13 Gruber O, Muller T, Falkai P: Dynamic interactions between neural systems underlying different components of verbal working memory. J Neural Transm 2007;114:1047-1050.

14 Goldman-Rakic PS: The prefrontal landscape: implications of functional architecture for understanding human mentation and the central executive. Philos Trans R Soc Lond B Biol Sci 1996;351:1445-1453.

15 Aboitiz F, Garcia VR: The evolutionary origin of the language areas in the human brain. A neuroanatomical perspective. Brain Res Brain Res Rev 1997;25:381-396.

16 D'Esposito M, Cooney JW, Gazzaley A, Gibbs SE, Postle BR: Is the prefrontal cortex necessary for delay task performance? Evidence from lesion and fMRI data. J Int Neuropsychol Soc 2006;12:248-260.

17 Price CJ, Friston KJ: Functional imaging studies of neuropsychological patients: applications and limitations. Neurocase 2002; 8:345-354.

18 Price CJ, Friston KJ: Degeneracy and cognitive anatomy. Trends Cogn Sci 2002;6:416421.

19 Henseler I, Gruber O, Kraft S, Krick C, Reith W, Falkai P: Compensatory hyperactivations as markers of latent working memory dysfunctions in patients with obsessive-compulsive disorder: an fMRI study. J Psychiatry Neurosci 2008;33:209-215.

20 Henseler I, Falkai P, Gruber O: A systematic fMRI investigation of the brain systems subserving different working memory components in schizophrenia. Eur J Neurosci 2009;30:693-702.

21 Gruber O, Tost H, Henseler I, Schmael C, Scherk H, Ende G, Ruf M, Falkai P, Rietschel M: Pathological amygdala activation during working memory performance: evidence for a pathophysiological trait marker in bipolar affective disorder. Hum Brain Mapp 2010; 31:115-125.
22 Lohmann G, Muller K, Bosch V, Mentzel H, Hessler S, Chen L, Zysset S, von Cramon DY: LIPSIA - A new software system for the evaluation of functional magnetic resonance images of the human brain. Comput Med Imaging Graph 2001;25:449-457.

23 Baldo JV, Dronkers NF: The role of inferior parietal and inferior frontal cortex in working memory. Neuropsychology 2006;20: 529-538.

24 D’Esposito M, Postle BR: The dependence of span and delayed-response performance on prefrontal cortex. Neuropsychologia 1999; 37:1303-1315.

25 Amassian VE, Maccabee PJ: Transcranial magnetic stimulation. Conf Proc IEEE Eng Med Biol Soc 2006;1:1620-1623.

-26 Jahanshahi M, Profice P, Brown RG, Ridding MC, Dirnberger G, Rothwell JC: The effects of transcranial magnetic stimulation over the dorsolateral prefrontal cortex on suppression of habitual counting during random number generation. Brain 1998;121: 1533-1544.

-27 Siebner HR, Hartwigsen G, Kassuba T, Rothwell JC: How does transcranial magnetic stimulation modify neuronal activity in the brain? Implications for studies of cognition. Cortex 2009;45:1035-1042.

-28 Siebner HR, Bergmann TO, Bestmann S, Massimini M, Johansen-Berg H, Mochizuki $\mathrm{H}$, Bohning DE, Boorman ED, Groppa S, Miniussi C, Pascual-Leone A, Huber R, Taylor PC, Ilmoniemi RJ, De Gennaro L, Strafella AP, Kahkonen S, Kloppel S, Frisoni GB, George MS, Hallett M, Brandt SA, Rushworth MF, Ziemann U, Rothwell JC, Ward N, Cohen LG, Baudewig J, Paus T, Ugawa Y, Rossini PM: Consensus paper: combining transcranial stimulation with neuroimaging. Brain Stimul 2009;2:58-80.

29 Vallar G, Di Betta AM, Silveri MC: The phonological short-term store-rehearsal system: patterns of impairment and neural correlates. Neuropsychologia 1997;35:795-812. 